\title{
Conscious sedation or local anesthesia during endovascular treatment for acute ischemic stroke
}

Rob A. van de Graaf, MD,* Noor Samuels, MD,* Maxim J.H.L. Mulder, MD, PhD, Ismail Eralp, MD, PhD, Adriaan C.G.M. van Es, MD, PhD, Diederik W.J. Dippel, MD, PhD, Aad van der Lugt, MD, PhD, and Bart J. Emmer, MD, PhD, for the Multicenter Randomized Clinical Trial of Endovascular Treatment of Acute Ischemic Stroke in the Netherlands (MR CLEAN) Registry Investigators

Neurology ${ }^{\circledR}$ 2018;91:e19-e25. doi:10.1212/WNL.0000000000005732

\section{Abstract}

\section{Objective}

To investigate the effect of conscious sedation (CS) on functional outcome and complication rates after intra-arterial treatment (IAT) for acute ischemic stroke (AIS) compared to the use of local anesthesia (LA) at the puncture site only.

\section{Methods}

Patients undergoing IAT for AIS with CS or LA in the Erasmus University Medical Center from March 2014 to June 2016 were included for analysis. The primary outcome was the score on the ordinal modified Rankin Scale (mRS). We compared CS to LA by ordinal logistic regression with covariate adjustment using propensity scoring.

\section{Results}

In 146 AIS patients treated with IAT, use of CS was associated with a shift towards worse mRS scores (odds ratio [OR] 0.4 [95\% confidence interval (CI) 0.2-0.7]) compared to LA. Mortality after 90 days was higher in the CS group compared to the LA group (OR 2.3 [95\% CI 1.0-5.2]). No differences between groups were noted with regard to procedure duration (8 minutes, $\beta=6.3[95 \% \mathrm{CI}-7.4$ to 20.0$]$ ) or occurrence of procedure-related complications (OR $1.3[95 \%$ CI $0.6-2.7])$.

\section{Conclusion}

CS was associated with poor functional outcome and increased mortality rates compared to LA. Furthermore, CS did not reduce duration of intervention or interventional complications. CS during IAT for AIS is of no benefit if LA is considered safe.

\section{Classification of evidence}

This study provides Class II evidence, because of nonrandom allocation, that for patients with AIS undergoing IAT, LA rather than CS improves functional outcome.

\author{
Correspondence \\ Dr. van de Graaf \\ r.a.vandegraaf@ \\ erasmusmc.nl
}

\section{RELATED ARTICLE}

\section{Editorial}

Optimal anesthetic strategy for endovascular stroke therapy: Begging for a good trial

Page 16

\section{MORE ONLINE}

\section{$\rightarrow$ Class of Evidence}

Criteria for rating therapeutic and diagnostic studies

NPub.org/coe

\section{ค Podcast}

Dr. Brian Eckerle talks with Dr. Rob van de Graaf about his paper on conscious sedation vs local anesthesia during endovascular treatment for AIS.

NPub.org/5113qo

\footnotetext{
*These authors contributed equally to this work.

From the Departments of Radiology (R.A.v.d.G., N.S., M.J.H.L.M., A.C.G.M.v.E., A.v.d.L., B.J.E.), Neurology (R.A.v.d.G., N.S., M.J.H.L.M., D.W.J.D.), and Anesthesia (I.E.), Erasmus MC, University Medical Center Rotterdam; and the Department of Radiology (B.J.E.), Academic Medical Center, Amsterdam, the Netherlands.

Go to Neurology.org/N for full disclosures. Funding information and disclosures deemed relevant by the authors, if any, are provided at the end of the article. 


\section{Glossary}

acOR = adjusted common odds ratio; AIS = acute ischemic stroke; $\mathbf{C I}=$ confidence interval; $\mathbf{C S}=$ conscious sedation; DSA = digital subtraction angiography; ED = emergency department; eTICI = modified thrombolysis in cerebral infarction including a $2 \mathrm{C}$ grade; $\mathbf{G A}=$ general anesthesia; $\mathbf{I A T}=$ intra-arterial treatment; $\mathbf{L A}=$ local anesthesia; MR CLEAN $=$ Multicenter Randomized Clinical Trial of Endovascular Treatment for Acute Ischemic Stroke in the Netherlands; mRS = modified Rankin Scale; $\mathbf{m T I C I}=$ modified thrombolysis in cerebral infarction; NIHSS $=$ NIH Stroke Scale.

Anesthetic support is commonly used during intra-arterial treatment (IAT) procedures for large vessel occlusions in acute ischemic stroke (AIS). ${ }^{1}$ The aim of anesthetic support during IAT is to reduce patient motion, increase patient comfort, facilitate fast treatment, and minimize the risk of complications. There are different options for anesthetic management during IAT; general anesthesia (GA), conscious sedation (CS), or local anesthesia (LA) at the puncture site only. CS is often considered as the ideal compromise during IAT by preserving patient cooperation, comfort, and procedural speed compared to LA, and reducing medication levels compared to GA. Although CS has become common practice during IAT, the effect on outcome is unknown. ${ }^{2}$ Post hoc analysis of the Multicenter Randomized Clinical Trialof Endovascular Treatment for Acute Ischemic Stroke in the Netherlands (MR CLEAN) data showed that GA had a negative influence on treatment effect of the intra-arterial procedure in AIS patients in comparison to non-GA (composite of LA and CS). ${ }^{3}$ This was also confirmed in the Highly Effective Reperfusion evaluated in Multiple Endovascular Stroke trials (HERMES) collaboration. ${ }^{4}$ On the other hand, recently published trials showed no advantage of CS on neurologic improvement after IAT compared to GA. ${ }^{5,6}$ We do not know of studies comparing GA or CS with LA at the groin puncture site only. Until now, it is unknown whether the use of CS during IAT has any positive influence on outcome, complications, or procedure times in AIS patients with a large intracranial vessel occlusion, when compared to LA. The aim of this study was to assess the effect of CS on functional outcome and occurrence of complications compared to LA.

\section{Methods}

\section{Classification of evidence}

We sought to answer the following research question: Does CS in patients with AIS caused by a large intracranial vessel occlusion of the anterior circulation improve functional outcome in comparison to LA? Class II level of evidence is assigned to this question.

\section{Data source and study population}

Patients who were enrolled in the MR CLEAN Registry were studied. The MR CLEAN Registry is a prospectively collected database containing all patients who underwent IAT for IAS in the Netherlands. The Registry started after the final MR CLEAN trial. All patients undergoing IAT (defined as at least entry into the angiography suite and arterial puncture) for acute ischemic stroke in the anterior and posterior circulation have been registered in the MR CLEAN Registry. For the current study, we restricted our analysis to patients treated in the Erasmus MC in Rotterdam from the start of the MR CLEAN Registry in March 2014 until June 2016. As our center was the only participating center in which the use of CS during IAT was standard care for a defined time period, we restricted our analysis to single center data to minimize selection bias. We additionally applied the following inclusion criteria: arterial puncture within 6.5 hours of symptom onset; age 18 years and older; intracranial proximal arterial occlusion in the anterior circulation (intracranial carotid artery or middle or anterior cerebral artery), demonstrated by CT angiography, magnetic resonance angiography, or digital subtraction angiography (DSA). We excluded patients with prestroke modified Rankin Scale (mRS) score higher than 2 points, when GA was performed as first line of defense, or when type of anesthesia was not documented. Study results are reported in accordance with the Strengthening the Reporting of Observational Studies in Epidemiology (STROBE) statement. ${ }^{7}$

\section{Anesthetic procedure}

The protocol in our institution stated that, if anesthetic support was available, CS should be started at the beginning of the IAT procedure. When CS could not be initiated due to unavailability of the attending anesthesiologist, the intervention team would go ahead and an attempt would be made to perform IAT directly under LA. If during the procedure it became apparent that IAT was not possible due to restlessness of the patient, the anesthesiology department would be requested to perform CS. The choice of anesthetic agents used for CS was left to the discretion of the anesthesiologist. Medication strategy was based on either propofol or remifentanil. Administered propofol doses ranged from 2 to 6 $\mathrm{mg} / \mathrm{kg} / \mathrm{h}$ and remifentanil doses from 1 to $4 \mu \mathrm{g} / \mathrm{kg} / \mathrm{h}$.

\section{Study outcomes}

The primary outcome was the mRS score (a 7-point scale ranging from 0 "no symptoms" to 6 "dead") at 90 days after IAT. Secondary outcomes included a score of 2 or less on the $\mathrm{mRS}$, indicating good functional outcome; death within 7, 30, and 90 days postintervention; and NIH Stroke Scale (NIHSS) score indicating neurologic deficit on a $0-42$ scale at $24-48$ hours postintervention. ${ }^{8,9}$ A higher NIHSS score indicates a more severe deficit. Procedure-related outcome measures included modified thrombolysis in cerebral infarction (mTICI) score on DSA, total procedure time, and procedure-related complications. The mTICI score ranges from 0 (no antegrade 
reperfusion of the occluded vascular territory) to 3 (complete antegrade reperfusion of the occluded vascular territory). For eTICI, grade 2C (slow flow in a few distal cortical vessels or presence of small distal cortical emboli, corresponding to $90 \%-99 \%$ reperfusion) was added to the original mTICI score. ${ }^{10}$ Procedure-related complications included vessel perforation, vessel dissection, new clot, distal thrombus, vasospasm, hemorrhage, and other. Procedure-related complications and eTICI score were assessed by core laboratory. Serious adverse events included symptomatic intracranial hemorrhage, progression of ischemic stroke, new ischemic stroke, pneumonia, other infections, cardiac ischemia, extracranial hemorrhage, allergic reactions, and other adverse events. Investigators who assessed primary and secondary outcomes, procedure-related outcome, and procedure-related complications were not aware of the type of anesthetic support during the procedure.

\section{Statistical methodology and procedures}

Based on the intention-to-treat principle, patients converted from LA to CS during the IAT procedure were included in the LA group. In case the anesthesia team was not available just before starting the IAT procedure and the IAT procedure seemed unsafe under LA (due to excessive movement), the anesthesia team was called immediately without starting the procedure to increase procedural safety. If the decision for anesthesia in the form of CS was made before groin puncture, the patient was included in the CS group. For variables with missing values in fewer than $5 \%$ of patients, we used single imputation by mean for continuous variables and by mode for categorical variables. Normality assessment of data was performed both visually and by means of the KolmogorovSmirnov test. One-way analysis of variance was used for parametric and Kruskal-Wallis test for nonparametric testing. Both categorical and dichotomous variables were tested using crosstabs and are shown as percentages.

Possible selection bias was addressed by performing adjustment for covariates by propensity score for both primary and secondary outcomes. Variables related to anesthetic management and outcome were selected based on clinical experience and previous literature. The saturated propensity model included the variables age, sex, previous stroke, diabetes mellitus, atrial fibrillation, hypertension, history of myocardial infarction, peripheral artery disease, prestroke mRS score, NIHSS at baseline, aphasia score, preinterventional eTICI score, and time from stroke onset to groin puncture. For each case, a propensity score was calculated using the propensity model. This propensity score, yielding the probability for a patient to receive anesthetic management in the form of CS given the baseline characteristics, was then incorporated in a regression model. Propensity score adjustment was performed by means of a logistic regression model for binary outcomes. The propensity score was then used in an ordinal logistic regression model to adjust the estimate of the effect of CS on the mRS score. This effect is expressed as an adjusted common odds ratio (acOR) with 95\% confidence interval $(\mathrm{CI})$, as the $\mathrm{mRS}$ is an ordered categorical outcome. A $p$ value of $<0.05$ was considered significant in all applied tests. All statistical analyses were performed with Stata 14.0 software (StataCorp, College Station, TX).

\section{Standard protocol approvals, registrations, and patient consents}

The MR CLEAN Registry was reviewed and approved by the medical ethics committee and research board of the Erasmus MC, University Medical Center, Rotterdam, the Netherlands (MEC-2014-235). This approval extends to all participating centers in the Netherlands. Study candidates received verbal and written explanation of the study and had the opportunity to opt out. Coded data were obtained and stored at Erasmus $\mathrm{MC}$, and scientific analyses were approved and supervised by a central writing committee. The MR CLEAN Registry study protocol is available on mrclean-trial.org/docs/latestprotocol.pdf.

\section{Data availability}

Data cannot be made available, as no patient approval has been obtained for sharing coded data. However, syntax files and output of statistical analyses (Stata 14.0) will be made available upon request.

\section{Results}

\section{Patient characteristics}

Between March 2014 and June 2016, 205 patients underwent IAT at the Erasmus MC. A total of 146 patients met the inclusion criteria (figure). Sixty patients (41\%) received CS and 86 patients (59\%) LA at the groin puncture site only during IAT. Patients treated with IAT under CS less often had a history of previous stroke than patients treated without CS $(1.7 \%$ vs $14.0 \% ; p=0.01)$ (table 1$)$.

Figure Flowchart of patients included from Erasmus Medical Center (Erasmus MC) in the Multicenter Randomized Clinical Trial of Endovascular Treatment for Acute Ischemic Stroke (MR CLEAN) registry from March 2014 to June 2016

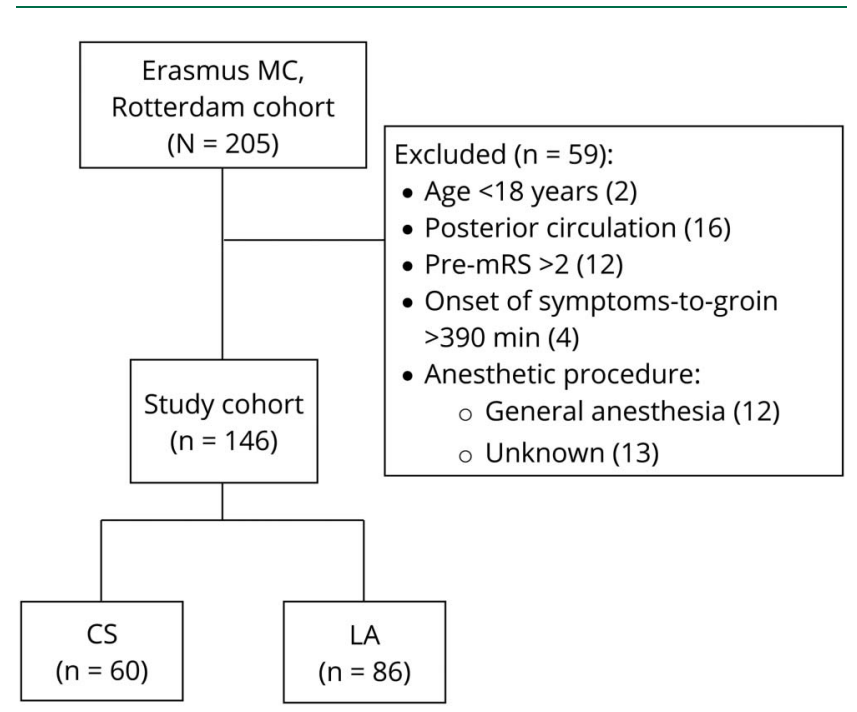

CS = conscious sedation; LA = local anesthesia; $m R S=$ modified Rankin Scale. 
Table 1 Baseline characteristics

CS $(n=60) \quad$ LA $(n=86) \quad p$ Value

\section{Patient characteristics}

Age, y, mean (SD)

Male sex, $\mathrm{n}(\%)$

$65.9(14.2)$

32 (53.3)

NIHSS, median (IQR)

$15(9.0-19)$

$69.2(13.5)$

0.16

Hemisphere, n (\%)

\begin{tabular}{|c|c|c|c|}
\hline Left & $22(44.9)$ & 30 (39.5) & 0.55 \\
\hline Right & $27(55.1)$ & $46(60.5)$ & 0.55 \\
\hline Systolic BP, mean (SD) & $152.7(27.0)$ & $151.3(24.0)$ & 0.74 \\
\hline Diastolic BP, mean (SD) & $81.7(16.7)$ & $82.7(15.6)$ & 0.72 \\
\hline IVT, n (\%) & $48(80.0)$ & $65(75.6)$ & 0.53 \\
\hline \multicolumn{4}{|l|}{ Medical history, n (\%) } \\
\hline Previous stroke & $1(1.7)$ & $12(14.0)$ & 0.01 \\
\hline Atrial fibrillation & $9(15.0)$ & $16(18.6)$ & 0.57 \\
\hline Hypertension & $28(47.5)$ & $48(55.8)$ & 0.32 \\
\hline Diabetes mellitus & $10(16.7)$ & $14(16.3)$ & 0.95 \\
\hline Myocardial infarction & $6(10.0)$ & $11(12.8)$ & 0.61 \\
\hline Peripheral arterial disease & $1(1.7)$ & $8(9.3)$ & 0.06 \\
\hline Prestroke mRS & & & 0.96 \\
\hline 0 & $52(92.9)$ & 78 (92.9) & \\
\hline 1 & $1(1.8)$ & $2(2.4)$ & \\
\hline 2 & $3(5.4)$ & $4(4.8)$ & \\
\hline
\end{tabular}

Imaging

Occluded segment, n (\%)

\begin{tabular}{|c|c|c|c|}
\hline M1 & $31(64.6)$ & $31(54.4)$ & 0.29 \\
\hline M2 & $12(25.0)$ & $19(33.3)$ & 0.35 \\
\hline ICA & $2(4.2)$ & $3(5.3)$ & 0.79 \\
\hline ICA-T & $3(6.3)$ & $4(7.0)$ & 0.88 \\
\hline Reperfusion before intervention (eTICI), n (\%) & & & 0.16 \\
\hline 0 & $45(79.0)$ & $62(80.5)$ & \\
\hline 1 & $9(15.8)$ & $6(7.8)$ & \\
\hline $2 A$ & $0(0)$ & $4(5.2)$ & \\
\hline 2B & $2(3.5)$ & $1(1.3)$ & \\
\hline $2 \mathrm{C} / 3$ & $1(1.8)$ & $4(5.2)$ & \\
\hline ASPECTS, median (IQR) & $8(7-10)$ & $9(8-10)$ & 0.05 \\
\hline \multicolumn{4}{|l|}{ Workflow } \\
\hline Time from stroke onset to IVT, min, median (IQR) & $70(55-111)$ & $72(55-87)$ & 0.89 \\
\hline Time from stroke onset to admission ED, ${ }^{a}$ min, median (IQR) & $166(126-210)$ & $138(101-189)$ & 0.06 \\
\hline Time from admission ED ${ }^{a}$ to groin puncture, min, median (IQR) & $55(35-79)$ & $54(37-72)$ & 0.98 \\
\hline
\end{tabular}


Table 1 Baseline characteristics (continued)

\begin{tabular}{lc}
\hline CS (n= 60) & LA (n = 86) \\
\hline Time from stroke onset to groin puncture, min, median (IQR) & $224.5(166-282.5)$ \\
\hline Abbreviations: ASPECTS = Alberta Stroke Program Early Computed Tomography Score; BP = blood pressure; CS = conscious sedation; ED = emergency \\
department; eTICI = modified thrombolysis in cerebral infarction including a 2C grade; ICA = internal carotid artery; ICA-T = internal carotid artery terminus; \\
IQR = interquartile range; IVT= IV thrombolysis; LA = local anesthesia at the groin puncture site; M(segment) = middle cerebral artery; NIHSS = NIH Stroke Scale. \\
Continuous data are presented as mean (SD) for normal distributed data or as median (IQR) for skewed data. \\
a Intervention center, Erasmus MC, Rotterdam.
\end{tabular}

\section{Logistics}

Time from stroke onset to admission to the emergency department (ED) of the Erasmus MC (intervention center) did not differ between the groups. Admission time was 166 minutes in the patients receiving CS vs 138 minutes for patients receiving LA, but this was not different $(p=0.06)$. Time from admission to the ED of the intervention center to groin puncture did not differ between groups (55 vs 54 minutes, $p=0.98$ ).

\section{Outcome}

Patients who underwent CS were more likely to have poor mRS scores at 90 days compared to LA (acOR 0.4 [95\% CI $0.2-0.7])$. Good functional outcome ( $\mathrm{mRS}$ score $\leq 2)$ at 90 days was less often seen in patients who underwent CS compared to LA (OR 0.4 [95\% CI 0.2-0.8]). Mortality within 30 days post IAT was higher in the CS group compared to the LA group (17/60 vs 10/86, OR 2.6 [95\% CI 1.0-6.4]). Also, mortality within 90 days post IAT was higher in the CS group, $35 \%(21 / 60)$ vs $16 \%(14 / 86)$ in the LA group (OR $2.3[95 \%$ CI 1.0-5.2]).

NIHSS at 24-48 hours post IAT was 12 in the patients receiving CS and 6 in the patients receiving LA, but not different ( $\beta 5.75$ [interquartile range -0.9 to 12.6$]$ ). Successful recanalization (eTICI $\geq 2 \mathrm{~B}$ ) was achieved in $72 \%(105 / 146)$ of all patients, without difference between the CS and LA group (OR 0.5 [95\% CI 0.2-1.0]). Successful recanalization at the eTICI $\geq 2 \mathrm{C}$ level on DSA was less often seen in the CS group (OR 0.4 [95\% CI 0.2-0.8]). There was no difference in time of stroke onset to reperfusion between the CS group and the LA group (284 vs 256 minutes, $\beta 10.7$ [95\% CI -14.0 to 35.3]). Also, total procedure time, procedure-related complications, and serious adverse events did not differ between groups (table 2). Conversion of anesthetic management was reported in 4 patients: twice from LA to CS, once from LA to GA, and once from CS to GA.

\section{Discussion}

In this cohort study, CS during IAT was associated with poor functional outcome and higher mortality compared to LA. Reperfusion rates, procedure duration, procedure-related complications, and serious adverse events did not differ between the 2 groups. Therefore, our results suggest that functional outcome is less influenced by LA than by CS as an anesthetic approach during IAT, bearing in mind that due to patient movement and need for procedural comfort the use of CS is sometimes inevitable.

Studies on anesthetic approaches during IAT focused only on GA compared to other types of anesthetic support (i.e., CS or non-GA) with contrasting results. ${ }^{4-6,11}$ Some suggested mechanisms present in both GA and CS may lead to worse outcome in comparison to LA. ${ }^{12,13}$ Delay in treatment initiation is considered an important disadvantage of GA, with effect on functional outcome. ${ }^{14}$ As "time is brain," 1 single hour of delay leads to a $6 \%$ decrease in good functional outcome. ${ }^{15}$ However, time from admission to the ED of the intervention center to groin puncture did not differ between patients who underwent IAT with CS compared to LA. In addition, patients undergoing CS have an increased risk of pulmonary aspiration, as they usually have not fasted before an IAT procedure. ${ }^{16}$ Other mechanisms potentially contributing to poor functional outcome after IAT may include the detrimental effect of periprocedural hypotension or the possible effects of anesthetic agents on the brain itself (i.e., direct neurotoxic). ${ }^{13,17-19}$

Time from stroke onset to admission to the ED of the intervention center was 28 minutes longer in the CS group, although not different from the LA group. This delay contributed to the longer time from stroke onset to reperfusion seen in the CS group. Notably, the net effect of CS on outcome remained, even with incorporating time from stroke onset to groin puncture in the propensity score. Time from admission to the ED of the intervention center to groin puncture was similar between patients who underwent IAT with CS or LA. Consequently, we were not able to detect a delay in the CS group regarding initiation of anesthetic management. Successful recanalization on eTICI $\geq 2 \mathrm{C}$ level was less often seen in the CS group. CS did not result in lower complication rates, contrary to the common belief that CS increases the safety of the procedure. Nonetheless, because of our relatively small sample size, these findings need to be confirmed in a larger prospective randomized study. Concerning our intention-to-treat principle for minimization of selection bias, the occurrence of conversion could not have influenced the results in favor of the LA group. 
Table 2 Effect of conscious sedation (CS) in patients undergoing intra-arterial treatment for acute ischemic stroke after propensity score adjustment

\begin{tabular}{|c|c|c|c|c|}
\hline & $\operatorname{CS}(n=60)$ & $\operatorname{LA}(n=86)$ & $\begin{array}{l}\text { Effect } \\
\text { measure }\end{array}$ & $\begin{array}{l}\text { Adjusted value } \\
(95 \% \mathrm{Cl})\end{array}$ \\
\hline \multicolumn{5}{|l|}{ Primary outcome } \\
\hline mRS at $90 \mathrm{~d}$, median (IQR) & $4(3-6)$ & $3(2-4)$ & acOR & $0.4(0.2$ to 0.7$)$ \\
\hline \multicolumn{5}{|l|}{ Secondary outcomes, clinical, n (\%) } \\
\hline $\mathrm{mRS} \leq 2$ at $90 \mathrm{~d}$ & $13(22)$ & $40(47)$ & OR & $0.4(0.2$ to 0.8$)$ \\
\hline Mortality at $7 \mathrm{~d}$ & $9(15)$ & $6(7)$ & OR & 1.8 (0.6 to 5.6$)$ \\
\hline Mortality at $30 \mathrm{~d}$ & $17(28)$ & $10(12)$ & OR & $2.6(1.0$ to 6.4$)$ \\
\hline Mortality at $90 \mathrm{~d}$ & $21(35)$ & $14(16)$ & OR & 2.3 (1.0 to 5.2$)$ \\
\hline NIHSS 24-48 h, median (IQR) & $12(7-19)$ & $6(4-14)$ & $\beta$ & $5.9(-0.9$ to 12.6$)$ \\
\hline \multicolumn{5}{|l|}{ Secondary outcome, radiologic } \\
\hline Reperfusion after intervention (eTICI $\geq 2 B$ ), $n(\%)$ & $38(63)$ & $67(78)$ & OR & 0.5 (0.2 to 1.0$)$ \\
\hline Reperfusion after intervention (eTICI $\geq 2 \mathrm{C}$ ), $\mathrm{n}(\%)$ & $23(38)$ & $53(62)$ & OR & $0.4(0.2$ to 0.8$)$ \\
\hline \multicolumn{5}{|l|}{ Secondary outcomes, time difference } \\
\hline Time from stroke onset to reperfusion, min, median (IQR) & $284(237-347)$ & $256(225-297)$ & $\beta$ & $10.7(-14.0$ to 35.3$)$ \\
\hline Duration of procedure, min, mean (SD) & $77(40)$ & $69(38)$ & $\beta$ & $6.3(-7.4$ to 20.0$)$ \\
\hline \multicolumn{5}{|l|}{ Secondary outcomes, safety measures, and serious adverse events } \\
\hline Procedure-related complications, $\mathrm{n}(\%)$ & $23(38)$ & $25(29)$ & OR & 1.3 (0.6 to 2.7 ) \\
\hline \multicolumn{5}{|l|}{ Serious adverse events, $\mathbf{n}(\%)$} \\
\hline Symptomatic ICH & $3(5)$ & $3(3)$ & OR & $1.7(0.3$ to 10.2$)$ \\
\hline $\mathrm{ECH}$ & $2(3)$ & $1(1)$ & OR & $2.4(0.2$ to 29.1$)$ \\
\hline Progression of stroke & $9(15)$ & $8(9)$ & OR & 1.3 (0.5 to 3.7 ) \\
\hline New ischemic stroke & $2(3)$ & $3(3)$ & OR & $0.8(0.1$ to 5.6$)$ \\
\hline Cardiac ischemia & 0 & $1(1)$ & OR & - \\
\hline Pneumonia & $12(20)$ & $9(10)$ & OR & 2.1 (0.8 to 5.8 ) \\
\hline Allergic reaction & 0 & 0 & OR & - \\
\hline Other infections & $2(3)$ & 0 & OR & - \\
\hline Other & $10(17)$ & $9(10)$ & OR & 1.4 (0.5 to 3.7$)$ \\
\hline
\end{tabular}

Abbreviations: acOR = adjusted common odds ratio; $\mathrm{Cl}=$ confidence interval; $\mathrm{ECH}=$ extracranial hemorrhage; eTICl = modified thrombolysis in cerebral infarction including a 2C grade; ICH = intracranial hemorrhage; LA = local anesthesia at the groin puncture site; mRS = modified Rankin Scale; NIHSS = NIH Stroke Scale; OR = odds ratio.

Variables in the propensity score model: age, sex, previous stroke, diabetes mellitus, atrial fibrillation, hypertension, history of myocardial infarction, peripheral artery disease, prestroke mRS score, NIHSS at baseline, aphasia score, preinterventional eTICl score, time from stroke onset to groin puncture. a Values of CS vs local anesthesia, adjusted by propensity score.

This study has several limitations. In this single-center study, patients were not randomized between CS and LA. Nevertheless, its observational design has the advantage that we observe the procedures in everyday practice, and the propensity score adjustment was performed to adjust for potential confounders between groups. Furthermore, results could have been confounded by variables not accounted for in the propensity model ("unmeasured confounding"). ${ }^{20}$ Confounding by indication might have been introduced, apart from protocol-based anesthetic management (CS), as the condition of the patient also influences the choice made by the intervention team. We tried to prevent this by saturating the propensity model. Regarding the baseline characteristics included in the propensity model, previous stroke and time from onset of stroke until groin puncture were distributed in disadvantage of CS. Another limitation is the lack of data on blood pressure during the IAT procedure. The generalizability of the results reported in this study is also limited by the 
lack of research on this topic and heterogeneity of IAT management between centers. Nevertheless, CS appears to influence outcome after IAT, which could be of relevance to physicians in the decision-making process for the most appropriate anesthetic management during IAT. Based on our results, a randomized trial evaluating outcome after IAT with LA, CS, or GA seems justified.

We found that CS is associated with poor functional outcome and higher mortality in patients who underwent IAT for AIS. Furthermore, CS did not reduce duration of intervention or procedure-related complications and did not increase reperfusion rates. Our results suggest that functional outcome is less influenced by LA than by CS as an anesthetic approach during IAT.

\section{Author contributions}

Rob A. van de Graaf: study concept and design, acquisition of data, analysis and interpretation of data, drafting of the manuscript. Noor Samuels: study concept and design, acquisition of data, analysis and interpretation of data, drafting of the manuscript. Maxim J.H.L. Mulder: study concept and design, acquisition of data, analysis and interpretation of data, critical revision of the manuscript for intellectual content. Ismail Eralp: study concept and design, critical revision of the manuscript for intellectual content. Adriaan C.G.M. van Es: study concept and design, critical revision of the manuscript for intellectual content. Diederik W.J. Dippel: study concept and design, analysis and interpretation of data, critical revision of the manuscript for intellectual content, study supervision. Aad van der Lugt: study concept and design, analysis and interpretation of data, critical revision of the manuscript for intellectual content, study supervision. Bart J. Emmer: study concept and design, analysis and interpretation of data, critical revision of the manuscript for intellectual content, study supervision.

\section{Acknowledgment}

The authors thank Hester Lingsma, $\mathrm{PhD}$, for statistical advice; and the MR CLEAN Registry Investigators. A list of all investigators is given in the appendix.

\section{Study funding}

No targeted funding reported.

\section{Disclosure}

R. van de Graaf, N. Samuels, M. Mulder, I. Eralp, and A. van Es report no disclosures relevant to the manuscript. D. Dippel: Erasmus MC received compensation from Stryker for activities of Dr. Dippel as a consultant and Bracco Imaging Ltd. for activities of Dr. Dippel as a consultant. In addition, Dr. Dippel is the recipient of unrestricted grants from Dutch Heart Foundation, Dutch Brain Foundation, Stryker, and Medtronic for the conduct of randomized trials of endovascular treatment for acute ischemic stroke. A. van der Lugt: Erasmus MC received compensation from Stryker for activities of Dr. van der Lugt as a consultant. In addition, Dr. van der Lugt is the recipient of unrestricted grants from Dutch Heart Foundation, Dutch Brain Foundation, Stryker, and Medtronic for the conduct of randomized trials of endovascular treatment for acute ischemic stroke. B. Emmer: Erasmus MC received compensation from Stryker for activities of Dr. Emmer as a consultant. Dr. Emmer is the recipient of compensation fees for review work from DEKRA and speaker fees from Novartis. Go to Neurology.org/N for full disclosures.

Received November 3, 2017. Accepted in final form March 23, 2018.

\section{References}

1. McDonagh DL, Olson DM, Kalia JS, Gupta R, Abou-Chebl A, Zaidat OO. Anesthesia and sedation practices among neurointerventionalists during acute ischemic stroke endovascular therapy. Front Neurol 2010;1:118.

2. Powers WJ, Derdeyn CP, Biller J, et al. 2015 American Heart Association/American Stroke Association focused update of the 2013 guidelines for the early management of patients with acute ischemic stroke regarding endovascular treatment: a guideline for healthcare professionals from the American Heart Association/American Stroke Association. Stroke 2015;46:3020-3035.

3. Berkhemer OA, van den Berg LA, Fransen PS, et al. The effect of anesthetic management during intra-arterial therapy for acute stroke in MR CLEAN. Neurology 2016;87:656-664.

4. Campbell BCV, van Zwam WH, Goyal M, et al. Effect of general anaesthesia on functional outcome in patients with anterior circulation ischaemic stroke having endovascular thrombectomy versus standard care: a meta-analysis of individual patient data. Lancet Neurol 2018;17:47-53.

5. Schonenberger S, Uhlmann L, Hacke W, et al. Effect of conscious sedation vs general anesthesia on early neurological improvement among patients with ischemic stroke undergoing endovascular thrombectomy: a randomized clinical trial. JAMA 2016; 316:1986-1996

6. Simonsen CZ, Yoo AJ, Sorensen LH, et al. Effect of general anesthesia and conscious sedation during endovascular therapy on infarct growth and clinical outcomes in acute ischemic stroke: a randomized clinical trial. JAMA Neurol 2018;75:470-477.

7. von Elm E, Altman DG, Egger M, et al. The Strengthening the Reporting of Observational Studies in Epidemiology (STROBE) statement: guidelines for reporting observational studies. Lancet 2007;370:1453-1457.

8. van Swieten JC, Koudstaal PJ, Visser MC, Schouten HJ, van Gijn J. Interobserver agreement for the assessment of handicap in stroke patients. Stroke 1988;19: 604-607.

9. Brott T, Adams HP Jr, Olinger CP, et al. Measurements of acute cerebral infarction: a clinical examination scale. Stroke 1989;20:864-870.

10. Noser EA, Shaltoni HM, Hall CE, et al. Aggressive mechanical clot disruption: a safe adjunct to thrombolytic therapy in acute stroke? Stroke 2005;36:292-296.

11. Lowhagen Henden P, Rentzos A, Karlsson JE, et al. General anesthesia versus conscious sedation for endovascular treatment of acute ischemic stroke: the AnStroke trial (anesthesia during stroke). Stroke 2017;48:1601-1607.

12. Anastasian $\mathrm{ZH}$. Anaesthetic management of the patient with acute ischaemic stroke. Br J Anaesth 2014;113(suppl 2):ii9-iil6.

13. Treurniet KM, Berkhemer OA, Immink RV, et al. A decrease in blood pressure is associated with unfavorable outcome in patients undergoing thrombectomy under general anesthesia. J Neurointerv Surg 2018;10:107-111.

14. Abou-Chebl A, Lin R, Hussain MS, et al. Conscious sedation versus general anesthesia during endovascular therapy for acute anterior circulation stroke: preliminary results from a retrospective, multicenter study. Stroke 2010;41:1175-1179.

15. Fransen PS, Berkhemer OA, Lingsma HF, et al. Time to reperfusion and treatment effect for acute ischemic stroke: a randomized clinical trial. JAMA Neurol 2016;73: 190-196.

16. American Society of Anesthesiologists Committee; Apfelbaum JL, Caplan RA, Connis RT, Epstein BS, Nickinovich DG, Warner MA. Practice guidelines for preoperative fasting and the use of pharmacologic agents to reduce the risk of pulmonary aspiration: application to healthy patients undergoing elective procedures: an updated report by the American Society of Anesthesiologists Committee on Standards and Practice Parameters. Anesthesiology 2011;114:495-511.

17. Claeys MA, Gepts E, Camu F. Haemodynamic changes during anaesthesia induced and maintained with propofol. Br J Anaesth 1988;60:3-9.

18. Fodale V, Schifilliti D, Pratico C, Santamaria LB. Remifentanil and the brain. Acta Anaesthesiol Scand 2008;52:319-326.

19. Whalin MK, Halenda KM, Haussen DC, et al. Even small decreases in blood pressure during conscious sedation affect clinical outcome after stroke thrombectomy: an analysis of hemodynamic thresholds. AJNR Am J Neuroradiol 2017;38:294-298.

20. Austin PC. An introduction to propensity score methods for reducing the effects of confounding in observational studies. Multivariate Behav Res 2011;46:399-424. 


\section{Neurology}

\section{Conscious sedation or local anesthesia during endovascular treatment for acute ischemic stroke}

Rob A. van de Graaf, Noor Samuels, Maxim J.H.L. Mulder, et al.

Neurology 2018;91;e19-e25 Published Online before print June 1, 2018

DOI 10.1212/WNL.0000000000005732

This information is current as of June 1, 2018

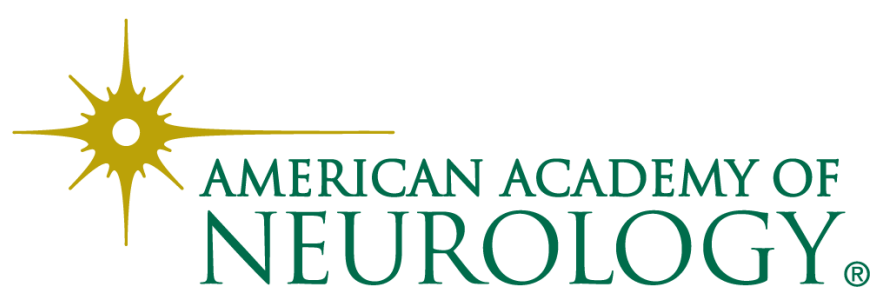




\section{Updated Information \& Services}

\section{Supplementary Material}

References

Citations

Subspecialty Collections

Permissions \& Licensing

\section{Reprints}

including high resolution figures, can be found at: http://n.neurology.org/content/91/1/e19.full

Supplementary material can be found at: http://n.neurology.org/content/suppl/2018/07/05/WNL.0000000000005 732.DC1

This article cites 20 articles, 8 of which you can access for free at: http://n.neurology.org/content/91/1/e19.full\#ref-list-1

This article has been cited by 3 HighWire-hosted articles: http://n.neurology.org/content/91/1/e19.full\#\#otherarticles

This article, along with others on similar topics, appears in the following collection(s):

\section{All Cerebrovascular disease/Stroke}

http://n.neurology.org/cgi/collection/all_cerebrovascular_disease_strok e

All Clinical Neurology

http://n.neurology.org/cgi/collection/all_clinical_neurology

\section{Class II}

http://n.neurology.org/cgi/collection/class_ii

Clinical trials Observational study (Cohort, Case control)

http://n.neurology.org/cgi/collection/clinical_trials_observational_stud y_cohort_case_control

Information about reproducing this article in parts (figures,tables) or in its entirety can be found online at:

http://www.neurology.org/about/about_the_journal\#permissions

Information about ordering reprints can be found online:

http://n.neurology.org/subscribers/advertise

Neurology ${ }^{\circledR}$ is the official journal of the American Academy of Neurology. Published continuously since 1951, it is now a weekly with 48 issues per year. Copyright () 2018 American Academy of Neurology. All rights reserved. Print ISSN: 0028-3878. Online ISSN: 1526-632X.

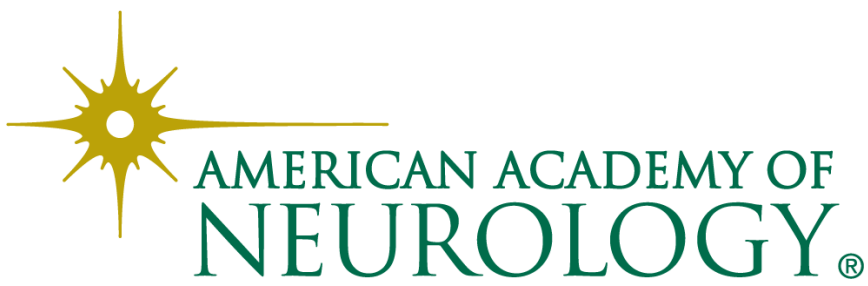

